\title{
Impact of Fixture Design on Sheet Metal Assembly Variation
}

\author{
Jaime A. Camelio and S. Jack Hu, Dept. of Mechanical Engineering, University of Michigan, \\ Ann Arbor, Michigan, USA \\ Dariusz Ceglarek, Dept. of Industrial and Systems Engineering, University of Wisconsin-Madison, \\ Madison, Wisconsin, USA
}

\begin{abstract}
This paper presents a new fixture design methodology for sheet metal assembly processes. It focuses on the impact of fixture position on the dimensional quality of sheet metal parts after assembly by considering the effect of part variation, tooling variation and assembly springback. An optimization algorithm combines finite element analysis and nonlinear programming methods to determine the optimal fixture position such that assembly variation is minimized. The optimized fixture layout enables significant reduction in assembly variation due to part and tooling variation. A case study is presented to illustrate the optimization procedure.
\end{abstract}

Keywords: Fixture Design, Dimensional Variation, Sheet Metal Assembly

\section{Nomenclature}

$F_{i} \quad$ Force applied by a position welding gun over part $i$

$L_{i} \quad$ Locator $i$

$\mathbf{K}_{\mathbf{a}} \quad$ Stiffness matrix of assembly ( $\mathrm{m} \times \mathrm{n}$ matrix)

$\mathbf{K}_{\mathrm{u}} \quad$ Stiffness matrix of parts (m×n matrix)

M Relocation matrix ( $\times \mathrm{n}$ matrix)

$\mathbf{M}_{\mathbf{i}} \quad$ Relocation matrix for part $i(\mathrm{n} \times \mathrm{n}$ matrix $)$

$R_{i} \quad$ Response force at locator $i$

$\mathbf{S}_{\mathbf{i}} \quad$ Sensitivity matrix for part i $(\mathrm{m} \times \mathrm{n}$ matrix $)$

$\mathbf{S}_{\mathrm{f}} \quad$ Sensitivity matrix for fixture errors $(\mathrm{m} \times \mathrm{q}$ matrix)

$\mathbf{V}_{\mathbf{a}}$ Assembly deviation after assembly process ( $\mathrm{m}$ vector)

$\mathbf{V}_{\mathbf{i}} \quad$ Part $i$ deviation (n vector)

$\mathbf{V}_{\mathbf{f}} \quad$ Fixture deviation (q vector)

$\mathbf{V}_{\mathbf{g}}$ Welding gun deviation ( $\mathrm{p}$ vector)

$\mathbf{V}_{\mathbf{s b}}$ Assembly springback for a welding gun error ( $m$ vector)

$\mathbf{V}_{\mathbf{u}} \quad$ Part or component initial deviation (n vector)

$\mathbf{V}_{\mathbf{u}}{ }^{\prime}$ Part deviation after the relocation process (n vector)

Originally published as paper no. DETC2002/DFM-34167, ASME 2002 Design Engineering Technical Conf. Permission granted by ASME for review and revision for publication in the Journal of Manufacturing Systems.

\section{Introduction}

Assembly processes are commonly used in the production of various consumer goods. Product manufacturing can be decomposed into two steps. First, components are fabricated using different methods such as machining, casting, injection molding,or metal forming. Second, these components are assembled or joined together using welding, riveting, fastening,or other joining methods. Fabrication processes are not perfect. They introduce variation in the components. In addition, assembly processes have their own variability. As a result, the dimensional quality of the final product is influenced by both component variation and assembly process variation. This paper focuses on variation analysis in sheet metal assemblies.

Several authors have proposed methodologies to predict variation in sheet metal assemblies (Jin and Shi 1999; Mantripragada and Whitney 1999; Ding, Ceglarek, and Shi 2000; Liu and $\mathrm{Hu}$ 1997; Rong, Ceglarek, and Shi 2000). They identified three sources of variation in such assemblies: component variation, fixture variation, and joining tool variation. Assuming rigid parts, Jin and Shi as well as Mantripragada and Whitney proposed a variation propagation model using state transition models. Ding, Ceglarek, and Shi (2000, 2002) developed a complete state-space modeling approach for dimensional control of in-plane rigid body motion of the assembly components. They considered two types of dimensional variation, part error and fixture error.

Considering the compliant nature of sheet metal parts, Liu and $\mathrm{Hu}$ presented a model to analyze the effect of component deviations and assembly springback on assembly variation by applying linear mechanics and statistics. The model considered the process at a station level. Using finite element methods (FEM), they constructed a sensitivity matrix for compliant parts of complex shapes. The sensi- 
tivity matrix established a linear relationship between the incoming part deviation and the output assembly deviation. Camelio, Hu, and Ceglarek (2003) extended this methodology to multistation assembly systems.

Fixtures play an important role in sheet metal assembly. In general, fixture elements can be classified by functionality into locators and clamps. Locators establish the datum reference frame and provide deterministic locating. Clamps provide total restraint by holding the part in position under the application of external forces during the manufacturing process. In sheet metal assembly, locators and clamps frequently coincide in the same position. In the remainder of this paper, the word fixture will be used to refer to both locators and clamps interchangeably.

One objective of fixture design is to determine the optimal layout of fixture elements such that fixture-workpiece deformation during clamping and processing is minimized provided that kinematic and total restraints are satisfied (Vallapuzha et al. 2002). Fixture design for machining processes has been widely studied (Lee and Haynes 1987; Menassa and DeVries 1991; Choudhuri and De Meter 1999; Kashyap and DeVries 1999; Sayeed and De Meter 1999). The traditional approach focuses on the determination of part and tooling deformation when an external force is applied over the workpiece. This force can be located at a fixed point or moving along the working surface.

Fixture design has been also studied for sheet metal assembly. Rearick, Hu, and Wu (1993) proposed an optimization algorithm to determine the optimal number and location of clamps required for deformable sheet metal parts. In 1996, Cai, Hu, and Yuan proposed new fixture principles for compliant sheet metal parts. They concluded that in order to locate and support compliant sheet metal parts, it is necessary to provide more than three locators in the primary plane due to part flexibility. Therefore, they proposed the $N$-2-1 fixture principle. In addition, they presented an optimization algorithm to find the optimal location of $N$ fixtures that minimizes the total workpiece deflection for a given external force. The
$\mathrm{N}$-2-1 optimal design was solved using nonlinear programming. The workpiece deformation was calculated using finite element methods.

Even though some authors have addressed fixture layout optimization for compliant sheet metal assembly, they have only studied the effect of external force disturbances and neglected the impact of fixture layout in the assembly process itself, which includes the interaction between tooling and part imperfections. Figure 1 gives a graphical representation of the fixture design problem solved in previous work (Rearick, Hu, and Wu 1993; Cai, Hu, and Yuan 1996) versus the fixture design approach proposed in this paper. In Figure la, the objective function minimizes part deformation under the application of a given force. The design variables are the fixtures' positions. In other words, fixtures are treated as supporting tools. Minimizing part deformation is important in a machining operation where material is removed; however, this may not be a significant factor for sheet metal assembly. In the assembly process illustrated in Figure $1 b$, the objective is to determine the impact of fixture position on the final assembly variation. In other words, how does the fixture position modify the contribution of part and tooling errors to the final assembly variation. In this case, fixtures are considered as sources of variation, and their placement may cause the transmitted variation to be different.

This paper focuses on the impact of fixture position (locating elements and holding elements) on the dimensional quality of sheet metal parts after assembly. The fixture layout is based on an $N-2-1$ locating principle. The optimization approach combines finite element analysis and nonlinear programming methods in finding the optimal fixture position that

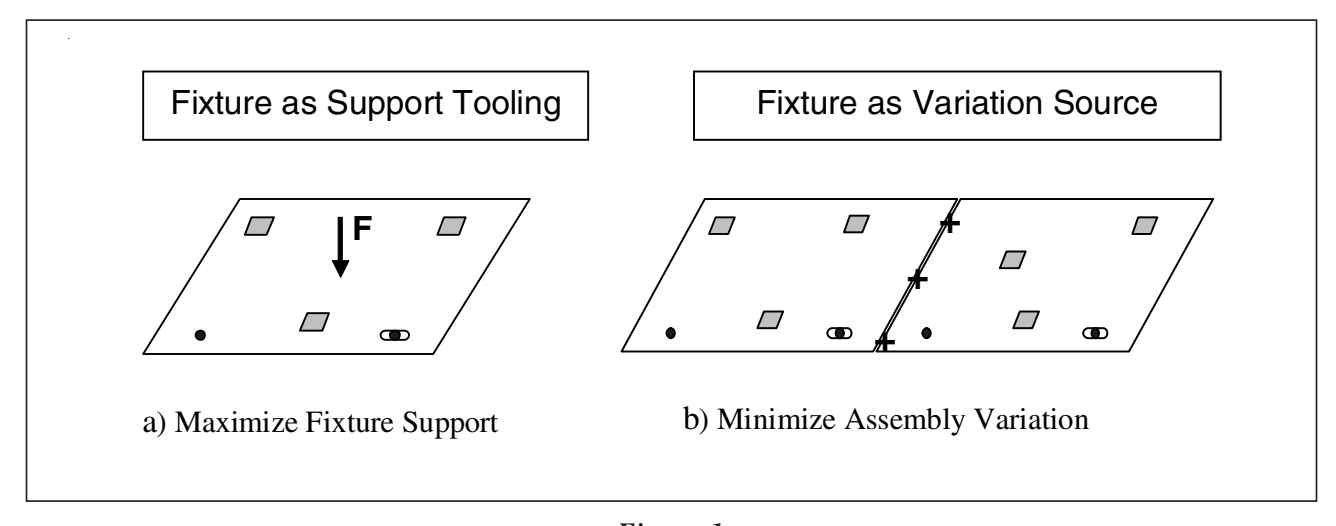

Figure 1

Problem Statement for Fixture Design 
minimizes assembly variation. The new objective function differs from that in previous work because it focuses on the assembly variation rather than on the component deflection for a given processing load.

In this paper, part variation and tooling variation are defined as the mean deviation, $\mu$, and the variance of the deviation, $\boldsymbol{\sigma}^{2}$. Part deviation is measured as the difference between the actual part and the nominal part at a specific point in a given direction. Part variation is only measured at key points, usually the points where the part will be joined and located. In this paper, part deviation is denoted as a vector $\mathbf{V} \in \mathbf{R}^{\mathrm{n} \times 1}$, the elements of which correspond to the deviation at each key characteristic point. Tooling variation is measured as the difference between the actual position of the tooling and its nominal or designed position. Therefore, a non-nominal or faulty fixture corresponds to a fixture that is forcing the part or assembly to a non-nominal position.

The next section presents a mechanistic model to calculate the system variation for a sheet metal assembly process, followed by the introduction of the effect of tooling position for different source of variation scenarios. Later sections present the optimization methodology, a case study, and conclusions.

\section{Assembly Model}

The assembly process is modeled based on the mechanistic variation simulation methodology developed by Liu and $\mathrm{Hu}$ (1997). This methodology assumes that: sheet metal deformation is in the linear elastic range; the material is isotropic; the fixture and weld gun are rigid; there is no thermal deformation; and the stiffness matrix remains constant for small part deformations. The joining method considered is resistance spot welding. As shown in Figure 2, the process is decomposed into four steps:

1. Parts are loaded in the assembly station (Figure $2 a$ )

2. Tooling is closed, deforming the parts to a nominal position (Figure $2 b$ )

3. Parts are assembled together (Figure 2c)

4. Tooling and extra locators are released and the assembly springs back (Figure $2 d$ )

The four steps of the sheet metal assembly process can be modeled as two sequential subprocesses. First, the locating process: the parts are loaded and located in the station. Second, the joining and

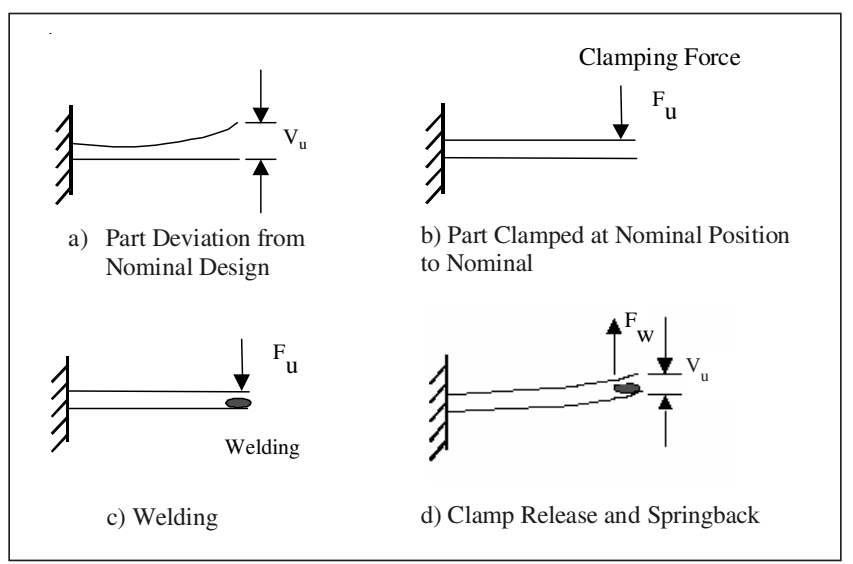

Figure 2

Sheet Metal Assembly Process (Camelio, Hu, and Ceglarek 2003)

springback processes: tooling is closed, parts are joined together, and the assembly springs back. Both processes will impact assembly variation. The assembly process is exemplified using a 2-D beam, but the same approach can be extended to 3-D.

\section{Locating Process}

Figure 3 shows the relocation effect on the primary plane for a 2-D beam. In the 2-D case, a 3-2-1 locating scheme assumes two (2) locators in the primary plane and one (1) locator in the secondary plane. In this example, part 1 is located using locators $\mathrm{P}_{1}$ and $\mathrm{P}_{2}$. Once the part is moved to another station, a new set of locators is utilized; then the input part variation for the joining process will be modified according to the new locators, $\mathrm{Q}_{1}$ and $\mathrm{Q}_{2}$.

Considering part out-of-plane deviation at points $\mathrm{Q}_{1}$ and $\mathrm{Q}_{2}$, the change in the coordinates of point $\mathrm{A}$ (part 1) due to relocating can be obtained using homogeneous transformations. Assuming small deviations, the displacements in the $x$ direction can be neglected. Therefore, the linear relationship of the part deviation in the $z$ direction (out-of-plane) due to reorientation will be as follows:

$$
\Delta V_{u}=\underbrace{\left[\begin{array}{cc}
\frac{V_{u}}{x_{Q_{2}}-x_{Q_{1}}}-1 & \frac{-V_{u}}{x_{Q_{2}}-x_{Q_{1}}}
\end{array}\right]}_{\mathbf{M}} \cdot\left[\begin{array}{c}
\Delta z_{Q_{1}} \\
\Delta z_{Q_{2}}
\end{array}\right]
$$

where $\mathbf{M}$ is the relocation matrix. Extending the same concept to different points on the part, the part deviation due to relocation can be written in a matrix form: 


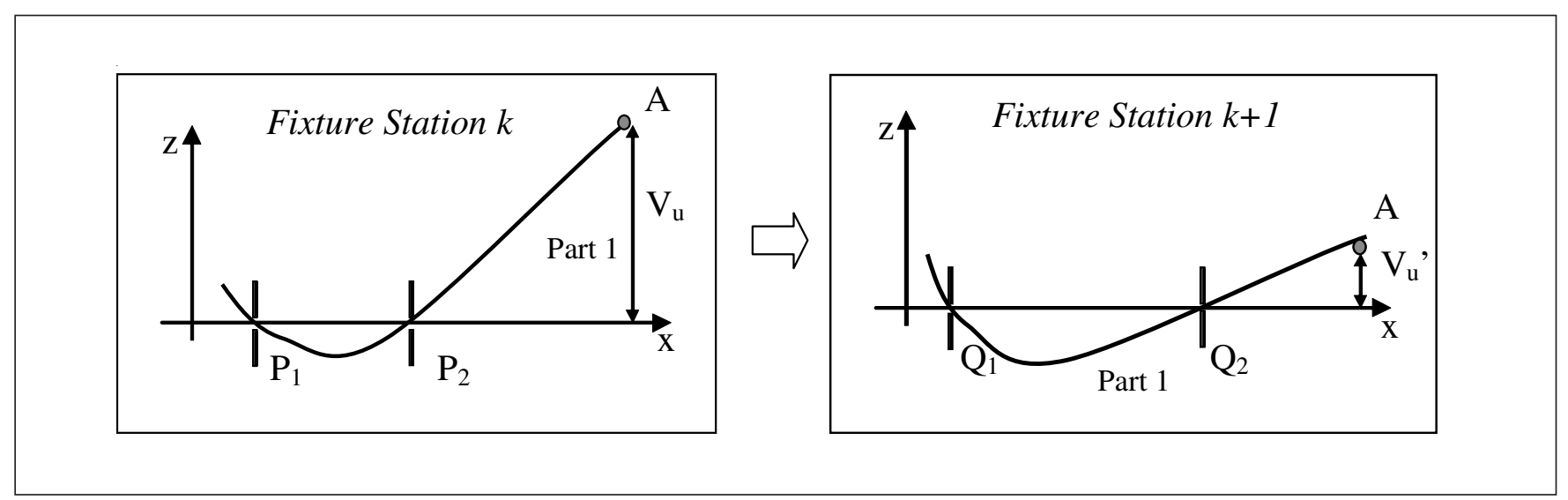

Figure 3

Locating Process from Station $(k)$ to $(k+1)$

$$
\mathbf{V}_{u}^{\prime}=V_{u}+M \cdot V_{u}=\bar{M} \cdot V_{u}
$$

where $\mathbf{V}_{\mathbf{u}}$ is the part deviation vector before locating, and $\mathbf{V}_{\mathbf{u}}{ }^{\prime}$ is the part deviation vector after the part has been located in the new station.

\section{Joining and Springback Process}

Based on the simulation method proposed in Liu and $\mathrm{Hu}$ (1997), the assembly deviation $\mathbf{V}_{\mathbf{a}}$ (Figure 2) can be determined knowing the input part variation, $\mathbf{V}_{\mathbf{u}}$, the components stiffness, $\mathbf{K}_{\mathbf{u}}$, and the assembly stiffness, $\mathbf{K}_{\mathbf{a}}$ [Eq. (2)].

$$
\begin{aligned}
& \mathbf{V}_{\mathrm{a}}=\left[\mathbf{K}_{\mathrm{a}}\right]^{-\mathbf{1}} \cdot \mathbf{K}_{\mathrm{u}} \cdot \mathbf{V}_{\mathbf{u}} \\
& \mathbf{S}=\left[\mathbf{K}_{\mathrm{a}}\right]^{-\mathbf{1}} \cdot\left[\mathbf{K}_{\mathbf{u}}\right] \\
& \mathbf{V}_{\mathrm{a}}=\mathbf{S} \cdot \mathbf{V}_{\mathbf{u}}
\end{aligned}
$$

where $\left[\mathbf{K}_{\mathbf{a}}\right]^{-1}$ is the inverse matrix of the assembly stiffness. The sensitivity matrix $\mathbf{S}$ [Eq. (3)] expresses the linear relation between the assembly deviation and the deviation for each source of variation. The sensitivity matrix $\mathbf{S}$ is determined by the method of influence coefficients (Liu and $\mathrm{Hu}$ 1997). This method calculates the component/part response to a unit force applied at each sources of variation using finite element methods. The inverse of these responses will be the stiffness of the component. Then, the corresponding assembly springback for a unit displacement in each source of variation is obtained. In other words, the method uses finite element methods (FEM) to indirectly calculate the stiffness of the assembly and components.
Finally, combining the relocation process [Eq. (1)] and the joining process [Eq. (4)], the assembly process variation for compliant parts can be obtained using:

$$
\mathbf{V}_{\mathrm{a}}=\mathbf{S} \cdot \overline{\mathbf{M}} \cdot \mathbf{V}_{\mathrm{u}}
$$

\section{Tooling Impact}

Part deviation is only one source of variation in sheet metal assembly. Tooling variation could also be an important contributor to assembly variation. Consequently, it is necessary to study the effect of tooling variation on the assembly variation. Tooling variation can be decomposed into two independent sources: weld gun variation and fixture variation, including clamps and locators. In this paper, two approaches are presented. First, tooling is considered to be perfect and the effect of fixture position is studied in the presence of part variation. Second, the impact of tooling variation and fixture position is analyzed.

\section{No Tooling Variation}

For simplicity, the impact of fixture position considering part variation is presented for a two-beam serial assembly. However, the analysis can be extended to 3-D. Parts are located using a set of locators, where the first locator $\left(\mathrm{L}_{1}\right.$ in part 1 and $\mathrm{L}_{4}$ in part 2) locates the part in the $x$ and $y$-directions and the second locator $\left(\mathrm{L}_{2}\right.$ in part 1 and $\mathrm{L}_{3}$ in part 2) constrains the part in the $y$-direction (Figure 4). As seen in Figure 4a, part 1 is not nominal. After joining both parts using spot welding (Figure $4 b$ ), some 


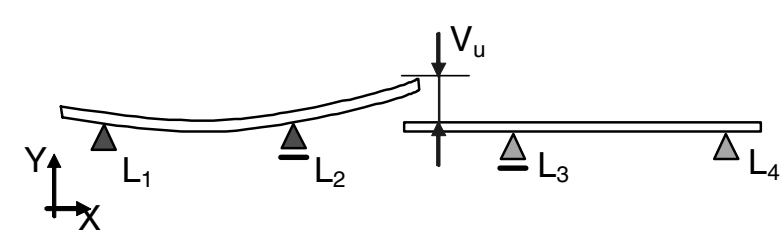

a) Part Variation

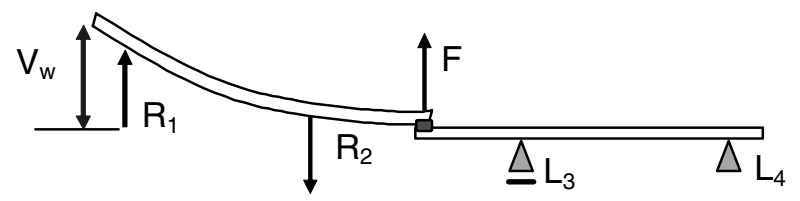

c) Assembly Springback for Case 1

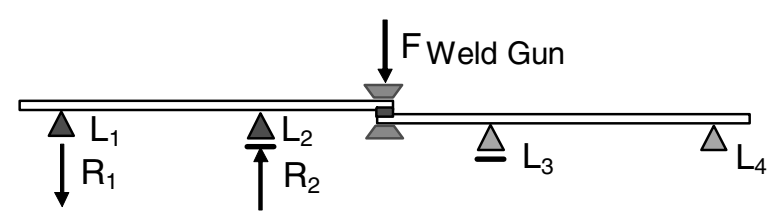

b) Welding gun pushes the part to nominal

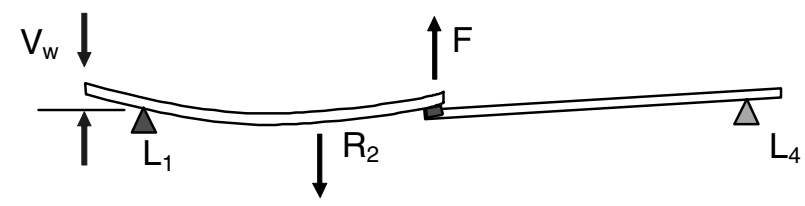

d) Assembly Springback for Case 2

Figure 4

No Tooling Variation Assembly Process

locators are released. In this analysis, two cases are presented:

Case 1: Part 1 is completely released after assembly (Figure $4 c$ ); that is, locators $\mathrm{L}_{1}$ and $\mathrm{L}_{2}$ are released.

Case 2: Part 1 and part 2 are not completely released after assembly; only locators $\mathrm{L}_{2}$ and $\mathrm{L}_{3}$ are released. Therefore, the assembly is overconstrained (Figure $4 d$ ).

It must be noted that the analysis presented is only valid for small deformations. Figure 4 shows amplified deviations in the $y$-direction. The apparently large part rotations are very small on a normal scale.

Using the assembly principle presented earlier in this paper, the assembly variation, for cases 1 and 2, can be calculated by Eq. (4). It must be noted that the sensitivity matrix, $\mathbf{S}$, changes for each case because the stiffness of the assembly, $\mathbf{K}_{\mathbf{a}}$, changes for each set of released locators. If we consider the assembly of two serial parts, Eq. (5) can be rewritten as follows:

$$
\begin{aligned}
& \mathbf{V}_{\mathrm{a}}=\left[\mathbf{K}_{\mathrm{a}}\right]^{-1} \cdot \mathbf{K}_{1} \cdot \mathbf{M}_{1} \times \mathbf{V}_{1} \\
& +\left[\mathrm{K}_{\mathrm{a}}\right]^{-1} \cdot \mathbf{K}_{2} \cdot \mathbf{M}_{2} \cdot \mathbf{V}_{2} \\
& \mathbf{V}_{\mathrm{a}}=\mathrm{S}_{1} \cdot \mathbf{M}_{1} \cdot \mathbf{V}_{1}+\mathrm{S}_{2} \cdot \mathbf{M}_{2} \cdot \mathbf{V}_{2}
\end{aligned}
$$

where $\mathbf{V}_{1}$ and $\mathbf{V}_{2}$ represent the input variations for each individual part, and $\mathbf{K}_{1}$ and $\mathbf{K}_{2}$ represent the stiffness of each part. $\mathbf{K}_{\mathbf{a}}$ is the stiffness matrix for the assembly after both beams are joined together. $\mathbf{M}_{1}$ and $\mathbf{M}_{2}$ are the relocation matrices.

To study the effect of fixture position on assembly variation, simulations were performed for different fixture positions. The assembly variation was calculated for different positions of fixture $\mathrm{L}_{2}$ on the $x$-axis. As expected, the stiffness of part 1 increased as fixture $\mathrm{L}_{2}$ is displaced away from fixture $\mathrm{L}_{1}$. In addition, the position of locator $\mathrm{L}_{2}$ modified the relocation matrix, $\mathbf{M}_{\mathbf{1}}$. The simulation results show that the increase in the stiffness of the part (matrix $\mathbf{S}$ ) when one of the locators is moved is compensated for the relocation effect (matrix $\mathbf{M}$ ).

Therefore, it can be concluded that in the scenario where there is no tooling error, but there is part deviation, the position of the locators does not impact the final assembly variation. In other words, assembly variation only depends on component deformations before assembly. This conclusion is valid for case 1 and case 2 .

In the past, several authors have studied optimal fixture design. The most common approach considers the support function of fixtures. In general, the fixture objective was to minimize the part deformation under the application of an external force (Menassa and DeVries 1991; Choudhuri and De Meter 1999; Cai, Hu, and Yuan 1996). However, this analysis showed that in absence of tooling error, sheet 
metal assembly variation is independent of fixture position. Moreover, support maximization can cause an unnecessary increase in component stiffness and may produce uncontrolled plastic deformation during the assembly process.

\section{Fixture Variation}

As stated previously in this paper, the sources of variation in sheet metal assembly process include part variation as well as tooling variation. In fact, Ceglarek and Shi $(1995,1996,1998)$ showed that the major root cause for auto body assembly process variation is tooling variation. This section analyzes the effect of fixture position and variation from fixture elements on assembly variation. The analysis is illustrated with a 2-D beam example. Again, the analysis can be extended to complex 3-D shapes using finite element methods.

Fixture variation can be decomposed into out-ofplane variation and in-plane variation. Out-of-plane variation is defined as the part or fixture deviation in the part's most flexible direction. In-plane variation is defined as the part or fixture variation that occurs in the plane normal to the direction of minimum stiffness. Generally, manufacturing forces are applied in the out-of-plane direction. In-plane forces can produce buckling.

In 1996, Cai, $\mathrm{Hu}$, and Yuan studied the influence of in-plane fixture variation. They assume that parts behaved as rigid bodies for displacement on locators in this plane. As seen in Figure 5, locator variation in the in-plane direction has a direct impact on the assembly variation. In this case, the assembly variation can be calculated using homogeneous transformation matrices. It can be intuitively deduced that for in-plane locator variation the optimal fixture location is obtained when the locators are as far apart as possible along the longer edge of the sheet metal (Cai, $\mathrm{Hu}$, and Yuan 1996). However, if the same fixture constrains the out-of-plane displacement, then the effect of fixture position in the direction normal to the surface should also be considered.

\section{Out-of-Plane Fixture Variation}

Out-of-plane fixture variation is defined as the fixture variation in the direction of flexible deformation. Using the mechanistic simulation model, presented earlier, the effect of fixture error on the assembly variation can be modeled using a method

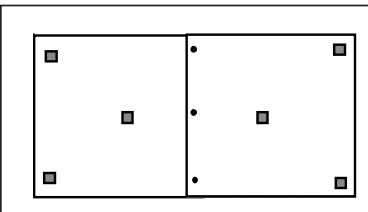

(a) Nominal Parts

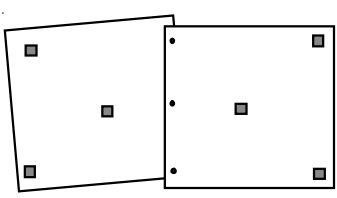

(b) Assembly Variation
Figure 5

In-Plane Fixture Variation

similar to that used to determine part variation. Therefore, using the method of influence coefficients (Liu and $\mathrm{Hu}$ 1997), the final variation for fixture variation, $\mathbf{V}_{\mathbf{f}}$, can be obtained using Eq. (8), where $\mathbf{S}_{\mathbf{f}}$ is the sensitivity matrix that relates the final assembly variation to an input fixture variation.

$$
\mathbf{V}_{\mathrm{a}}=\mathbf{S}_{\mathrm{f}} \cdot \mathbf{V}_{\mathrm{f}}
$$

The sensitivity matrix, $\mathbf{S}_{\mathbf{f}}$, is calculated by subtracting the assembly springback deformations from the part deformed by the tooling error.

This linear relationship is valid as long as we assume small deformations. In addition, for small deformations, the effect of part variation and fixture variation can be superposed. Then, the final variation combining both effects is as follows:

$$
\mathbf{V}_{\mathrm{a}}=\mathrm{S}_{1} \cdot \mathrm{M}_{1} \cdot \mathrm{V}_{1}+\mathrm{S}_{2} \cdot \mathrm{M}_{2} \cdot \mathrm{V}_{2}+\mathrm{S}_{\mathrm{f}} \cdot \mathbf{V}_{\mathrm{f}}
$$

The same example of a two-beam serial assembly is used for out-of-plane fixture analysis (Figure 6). Assuming that locator $\mathrm{L}_{2}$ has an error $\mathbf{V}_{\mathbf{f}}$, part 1 will be rotated about the $z$-axis (Figure $6 a$ ). The error $\mathbf{V}_{\mathbf{f}}$ produces a gap, $\mathbf{V}_{\mathbf{u}}$, between parts 1 and 2 that must be closed by the welding gun before assembly. In addition, the presence of a non-nominal fixture will interfere with the part when the welding gun is closed to its nominal position (Figure 6b). After the parts are joined together and the fixtures are released, the assembly springs back from this non-nominal position to a new position (Figures $6 c$ and $6 d$ ). In this analysis, two cases are presented:

Case 3: Part 1 is completely released after assembly (Figure 6c); that is, locators $\mathrm{L}_{1}$ and $\mathrm{L}_{2}$ are released.

Case 4: Part 1 and part 2 are not completely released after assembly; only locators $\mathrm{L}_{2}$ and $\mathrm{L}_{3}$ are released. Therefore, the assembly is overconstrained (Figure 6d). 


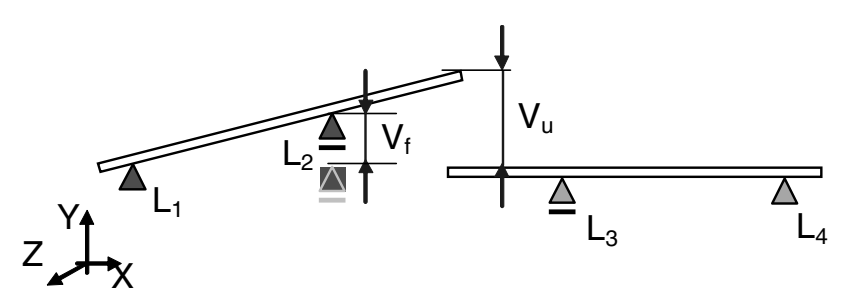

a) Part Rotation due to Fixture Error

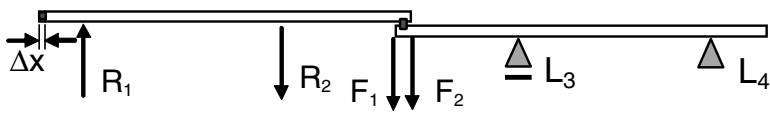

c) Assembly Springback for Case 3

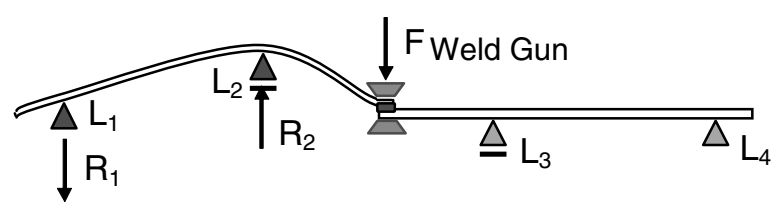

b) Welding gun pushes the part to nominal

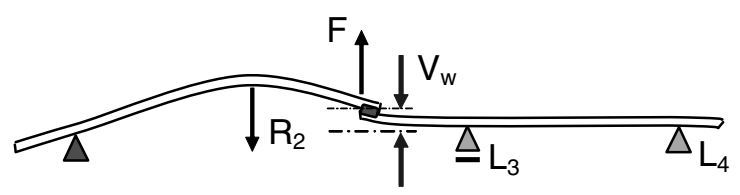

d) Assembly Springback for Case 4

Figure 6

Out-of-Plane Fixture Variation

As in the case of part variation, this analysis is used to estimate the impact of the locators' positions on the final assembly variation. Applying the method of influence coefficients for different positions of locator $\mathrm{L}_{2}$ along the $x$-axis, the sensitivity matrices $\mathbf{S}_{\mathbf{f}}$ can be obtained. Figure 7 shows $\mathbf{S}_{\mathbf{f}}$ for different positions of $\mathrm{L}_{2}$. In these cases, locator $\mathrm{L}_{2}$ had an offset of $1 \mathrm{~mm}$. As seen in Figure 7, the final assembly variation increases as locator $\mathrm{L}_{2}$ is moved closer to the welding gun position or to the nominal locator $\mathrm{L}_{1}$. Because the function is convex, there is an optimal position for $\mathrm{L}_{2}$ that minimizes the final assembly variation due to fixture error.

The effect that fixture error has on the assembly variation can be explained by the gap between part 1 and part 2. This gap increases when the failure locator is moved closer to another locator. When this gap is closed by the welding gun, a relative displacement between part 1 and part 2 on the $x$-direction is produced. The same phenomenon occurs when the fixture is moved closer to the welding gun and the part is forced to the nominal position before welding. Therefore, if the assembly is overconstrained (Figure 6d), the relative displacement between part 1 and 2 will increase the separation between the locating holes in both parts. As a result, the assembly will not be able to return to its nominal position. This effect produces an assembly deviation $\mathbf{V}_{\mathbf{a}}$. On the other hand, if the assembly is not overconstrained, the assembly is still longer than the distance between

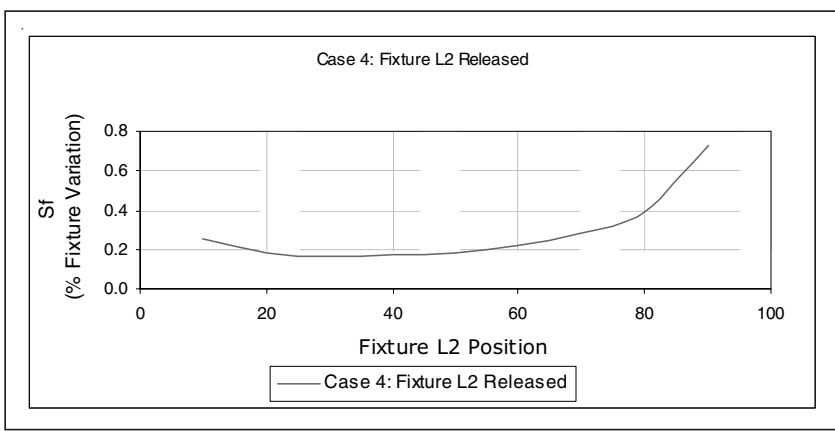

Figure 7

Assembly Sensitivity for Different Fixture Positions Considering Fixture Variation

$\mathrm{L}_{1}$ and $\mathrm{L}_{4}$, but it will return to its nominal position in the $y$-direction. The relative displacement in the $x$ direction cannot be observed using linear static beam theory; nonlinear geometry theory should be applied (NLGEOM in MSC/NASTRAN).

Consequently, in the presence of tooling error caused by a locator offset in the out-of-plane direction, the position of the fault locator or clamp will influence the assembly variation. This conclusion is valid when the resulting assembly is overconstrained, Case 4 (Figure $6 d$ ). In general, to minimize the effect of fixture variation, the faulty fixture should be moved as far from the welding locations as well as other fixtures as possible. An exact optimal position can be determined. In contrast, if the assembly is not overconstrained, the tooling error will not affect the assembly variation in the out-of-plane direction, 
Case 3 (Figure 6c). However, the assembly will be longer than nominal in the $x$-direction.

\section{Welding Gun Variation}

Liu and $\mathrm{Hu}$ (1995) demonstrated that welding gun variation has a large impact on the final assembly variation. The influence on assembly variation will depend on the type of weld gun. In general, the three types of weld guns used in industry for sheet metal assembly are: position-controlled weld gun, equalized weld gun, and force-controlled weld gun. In this paper, the model presented is based on the position-controlled weld gun.

A position-controlled weld gun is used to weld two parts to a nominal position. The position-controlled weld gun model assumes that the weld gun can apply a sufficient force to close the gap between the deviated part and its nominal position. As shown in Figure 8, part 1 has a deviation of $\mathbf{V}_{\mathbf{1}}$ and part 2 has a deviation of $\mathbf{V}_{2}$. In addition, the weld gun has a deviation, $\mathbf{V}_{\mathbf{g}}$, from nominal. The force required to close the gap in part 1 and 2 will be:

$$
\begin{aligned}
& F_{1}=K_{1}\left(V_{1}-V_{g}\right) \\
& F_{2}=K_{2}\left(V_{2}-V_{g}\right)
\end{aligned}
$$

where $\mathbf{K}_{\mathbf{1}}$ and $\mathbf{K}_{\mathbf{2}}$ are the stiffness matrices for part 1 and part 2, respectively.

The resulting force, $F=F_{1}+F_{2}$, will produce springback over the assembly. If $\mathbf{K}_{\mathbf{a}}$ is the stiffness of the assembly, the resulting springback will be

$$
\begin{aligned}
& \left.\mathbf{V}_{\mathrm{sb}}=\left[\mathrm{K}_{\mathrm{a}}\right]^{-1} \times \mathbf{F}=\left\{[\mathrm{K}]^{-1} \cdot \mathrm{K}_{1}\right]\right\} \cdot \mathbf{V}_{1}+\left\{\left[\mathrm{K}_{\mathrm{a}}\right]^{-1}\right. \\
& \left.\left.\cdot \mathbf{K}_{2}\right]\right\} \cdot \mathbf{V}_{2}-\left\{\left[\mathrm{K}_{\mathrm{a}}\right]^{-1} \cdot\left(\mathrm{K}_{1}+\mathrm{K}_{2}\right)\right\} \cdot \mathbf{V}_{\mathrm{g}}
\end{aligned}
$$

where $\mathbf{V}_{\text {sb }}$ represents the assembly springback effect contributed by the welding gun error. The final assembly variation will be the sum of the welding gun deviation and its springback.

$\mathbf{V}_{\mathrm{a}}=\mathrm{V}_{\mathrm{g}}+\left(\mathrm{S}_{1} \cdot \mathrm{V}_{1}+\mathrm{S}_{2} \cdot \mathrm{V}_{2}-\left(\mathrm{S}_{1}+\mathrm{S}_{2}\right) \cdot \mathrm{V}_{\mathrm{g}}\right)$

Equation (10) can be rewritten including the relocation effect in the case that the tooling location is changed. Therefore, the part variation, $\mathbf{V}_{\mathbf{i}}$, will be replaced by $\mathbf{M}_{\mathbf{i}} \cdot \mathbf{V}_{\mathbf{i}}$.

$$
\begin{aligned}
& \mathbf{V}_{\mathrm{a}}=\mathbf{S}_{1} \cdot \mathbf{M}_{1} \cdot \mathbf{V}_{1}+\mathbf{S}_{2} \cdot \mathbf{M}_{2} \cdot \mathbf{V}_{2}+\left(1-\mathbf{S}_{1}-\mathbf{S}_{2}\right) \cdot \mathbf{V}_{\mathrm{g}} \\
& \mathbf{S}_{\mathrm{g}}=\left(1-\mathbf{S}_{1}-\mathbf{S}_{2}\right)
\end{aligned}
$$

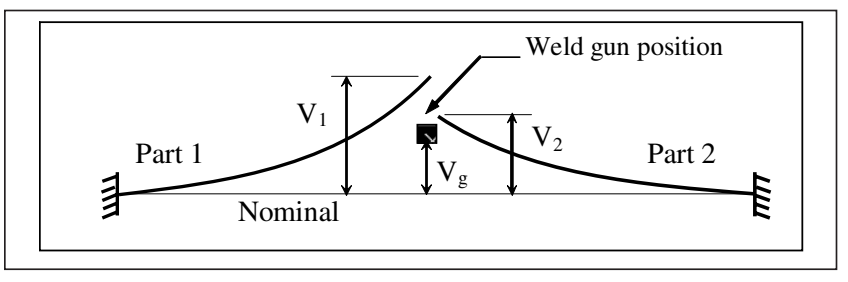

$$
\begin{gathered}
\text { Figure } 8 \\
\text { Impact of Position Weld Gun Variation } \\
\text { (Camelio, Hu, and Ceglarek 2003) }
\end{gathered}
$$

The sensitivity matrix for the welding gun variation is defined in Eq. (11). This result coincides with the "SUM ONE" property presented by Liu and $\mathrm{Hu}$ (1995). This principle states that the summation of the sensitivity coefficients of all part deviations and the tooling deviations must equal one.

The welding gun analysis is extended to include the influence of locator position in the presence of welding gun error. The same two-beam example is presented. Figure $9 a$ shows two nominal beams that are welded together in serial. A welding error, $\mathbf{V}_{\mathbf{g}}$, forced both parts to move to a non-nominal position (Figure 9b). In this analysis, two cases are presented:

Case 5: Part 1 is completely released after assembly (Figure $9 c$ ); that is, locators $\mathrm{L}_{1}$ and $\mathrm{L}_{2}$ are released.

Case 6: Part 1 and part 2 are not completely released after assembly; only locators $\mathrm{L}_{2}$ and $\mathrm{L}_{3}$ are released. Therefore, the assembly is overconstrained (Figure 9d).

Figure 10 shows welding gun sensitivity, $\mathbf{S}_{\mathrm{g}}$, for different fixture positions. $\mathbf{S}_{\mathrm{g}}$ is calculated for different positions of locators $\mathrm{L}_{2}$ and $\mathrm{L}_{3}$ along the $x$-axis. The positions of $\mathrm{L}_{2}$ and $\mathrm{L}_{3}$ are defined as the distances from locator $\mathrm{L}_{1}$ and $\mathrm{L}_{4}$, respectively. From the figure, it can be seen that the final assembly variation increases as the locators are moved closer to the welding gun locations.

As a result, in the presence of welding gun errors, the assembly variation is affected by fixture positions. This conclusion is valid if the assembly is overconstrained, Case 6 (Figure 9d). In this case, fixtures should be moved as far from the non-nominal welding guns as possible. Moreover, the analysis shows that the optimal fixture position is obtained when the fixture is located such it does not provide any support during the assembly process (zero stiffness). However, this solution is not feasible in a practical assembly process. Parts must be held in a 


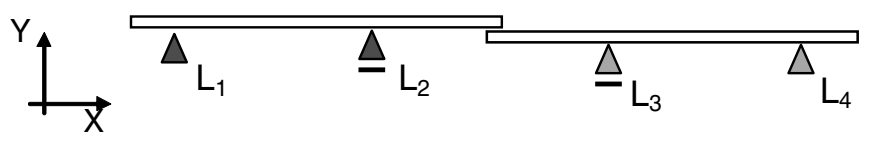

a) No Part Variation

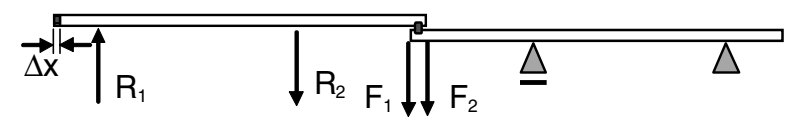

c) Assembly Springback for Case 5

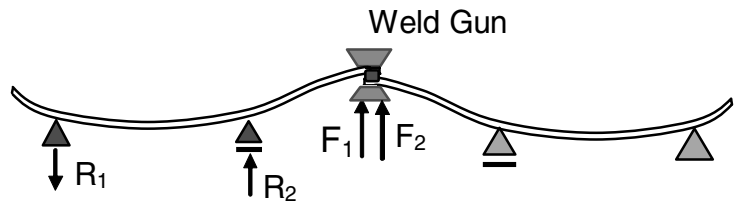

b) Welding gun pushes the part

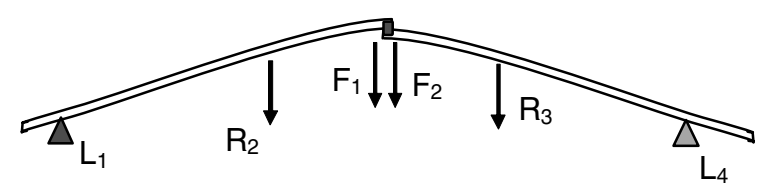

d) Assembly Springback for Case 6

Figure 9

Fixture Design Considering Welding Gun Variation

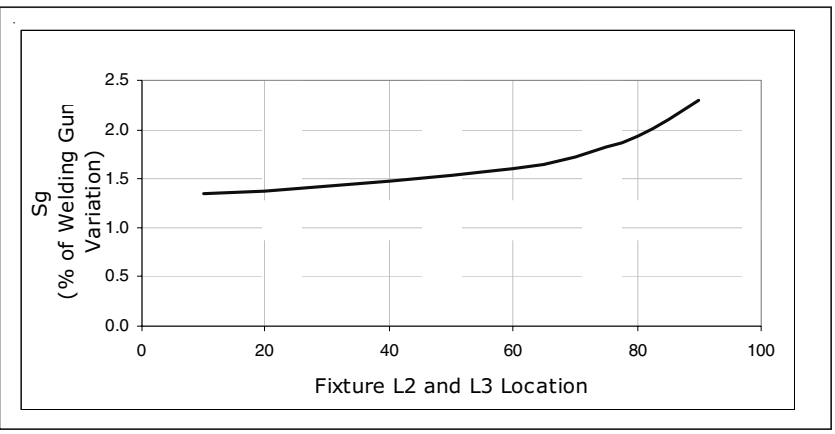

Figure 10

Assembly Variation for Different Fixture Position Considering Welding Gun Variation

specific position before assembly. On the other hand, assuming that parts are perfectly elastic and are not overconstrained, welding gun error will have no effect on the assembly dimensions in the out-of-plane direction, Case 5 (Figure 9c).

\section{Optimal Fixture Design}

The results presented in the previous section suggest that it is possible to find an optimal fixture position that minimizes the assembly variation given part and tooling variation. Combining the methodologies outlined in the different cases, and assuming small deformations, we can estimate the assembly variation of two serial parts as follows:

$$
\begin{aligned}
V_{\mathrm{a}} & =S_{1} \cdot M_{1} \cdot V_{1}+S_{2} \cdot M_{2} \cdot V_{2} \\
& +S_{g} \cdot V_{g}+S_{f} \cdot V_{f}
\end{aligned}
$$

where the values of $\mathbf{S}_{1}, \mathbf{S}_{2}, \mathbf{S}_{\mathfrak{t}}, \mathbf{S}_{\mathrm{g}}, \mathbf{M}_{1}$, and $\mathbf{M}_{\mathbf{2}}$ are function of the fixtures position. Then the assembly variation can be defined as a function of the fixtures position.

The objective function is defined as the sum of squares of the deviations of a set of key characteristic points on the assembly. In other words, the total assembly variation is defined as the sum of squares of the elements of the vector $\mathbf{V}_{\mathbf{a}}$. Due to the use of finite element methods (FEM), the mesh of each part must be defined such that each key point coincides with a node in the mesh. Therefore, the minimization problem to select the optimal fixture position can be written as:

Minimize

$$
F(\mathbf{X})=\sum_{i=1}^{n} V_{a_{i}}(\mathbf{X})^{2}
$$

Subject to

$$
\mathrm{G}(\mathbf{X}) \leq 0
$$

where $F(\mathbf{X})$ is the objective function of the assembly variation for the locator position vector, $\mathbf{X} . \mathbf{V}_{\mathbf{i}}(\mathbf{X})$ is the out-of-plane assembly deflection of the key characteristic point $i$ for a given fixture position vector $\mathbf{X}$. The only constraint imposed over the fixture location $(\mathbf{X})$ is that the fixtures must be located over the surface of the sheet metal part. Therefore, the inequality constraint $G(\mathbf{X})$ is defined such as the position of the fixtures must be in a given range.

The proposed methodology combines the use of the method of influence coefficients, finite element methods, and nonlinear programming (Figure 11). 


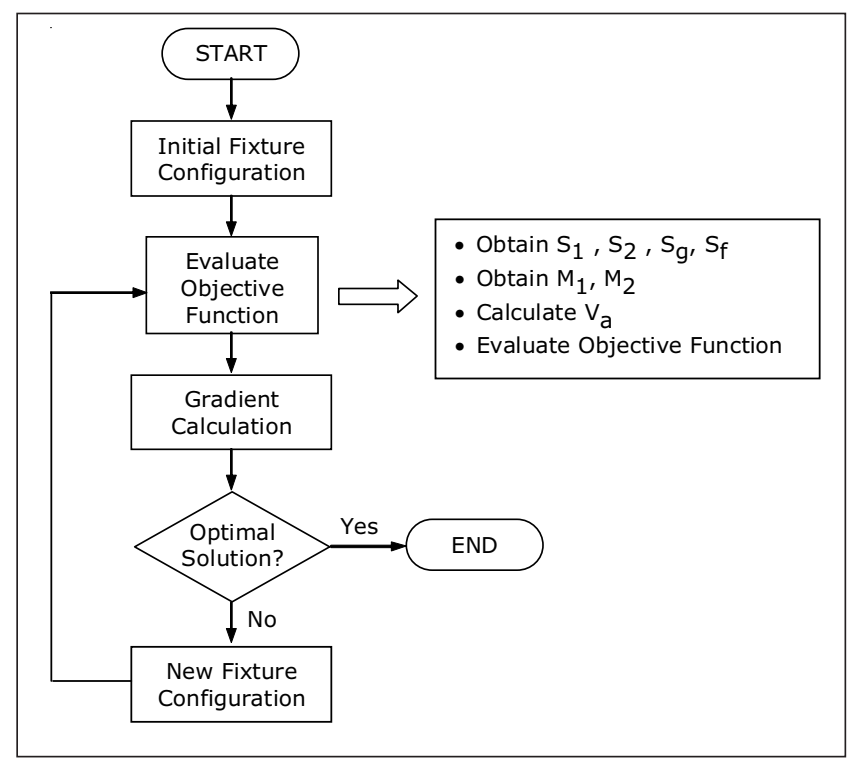

Figure 11

Optimal Fixture Position Flowchart

For complex part shapes, there is no analytical relation between $F(\mathbf{X})$ and $\mathbf{X}$, and it is necessary to use finite element methods (FEM) to calculate the assembly variation. Although, Eq. (12) is linear for the input variation, the sensitivity matrices $\left(\mathbf{S}_{\mathbf{1}}, \mathbf{S}_{\mathbf{2}}, \mathbf{S}_{\mathbf{f}}\right.$, and $\mathbf{S}_{\mathbf{g}}$ ) must be obtained for each different fixture position vector $\mathbf{X}$ using FEM. Due to the lack of a close form function for $F(\mathbf{X})$, the objective function gradient vector, $\mathbf{g}$, can be approximated using finite difference method:

$$
g_{i}=\frac{F\left(\mathbf{X}+\Delta \mathbf{X}_{i}\right)-F(\mathbf{X})}{\left\|\Delta \mathbf{X}_{i}\right\|}
$$

where $\Delta \mathbf{X}_{i}$, defined as $\Delta \mathbf{X}_{i}=$

$\left[\begin{array}{lllll}0 & \ldots & \delta_{i} & \ldots & 0\end{array}\right]^{\mathrm{T}}$, is a perturbation vector of the design variable $\mathbf{X}$ in the $i$-direction.

As presented by Rearick, $\mathrm{Hu}$, and $\mathrm{Wu}$ (1993), the FEM discrete mesh produces some optimization difficulties each time the fixture position vector is redefined. Specifically, the main problem is that NLP algorithms can localize fixtures at any continuous position over the geometric space, but the mesh model only has defined some discrete position over the part surface. Therefore, a remeshing algorithm is necessary to redefine the nodes so that the fixtures can be located in the model. Cai, Hu, and Yuan (1996) proposed the use of multipoint constraint (MPC) to avoid remeshing the FEA model. In the optimization algorithm presented here, both concepts are necessary to calculate the assembly variation for different fixture positions. Multipoint constraint (MPC) is used to calculate the sensitivity matrices $\mathbf{S}_{\mathbf{1}}$ and $\mathbf{S}_{2}$. However, MPC cannot be used to calculate the fixture sensitivity matrix, $\mathbf{S}_{\mathrm{t}}$, because during the analysis with FEA it is necessary to apply a unit force over the moving fixture and MPC is limited to displacement constraints. Therefore, the model must be remeshed to obtain $\mathbf{S}_{\mathbf{t}}$.

\section{Application Example}

In this section, the optimization methodology is illustrated by an example. Two identical sheet metal parts are joined together in one station, as shown in Figure 12. Each part has dimensions $200 \times 100 \times 1$ $\mathrm{mm}^{3}$. The material is mild steel with Young's modulus $\mathrm{E}=207,000 \mathrm{~N} / \mathrm{mm}^{2}$ and Poisson ratio $v=0.3$. Three welds $\left(\mathrm{W}_{1}, \mathrm{~W}_{2}\right.$, and $\left.\mathrm{W}_{3}\right)$ are used to join the parts. A 3-2-1 locating scheme is used to locate each part. The design variable considered is the position of locator $P$ on part 1. Locator $P$ constrains the part displacement in the $z$-direction (out-of-plane).

The sensitivity matrices are obtained using the FEM software ANSYS. The optimization algorithm used is the constrained nonlinear programming function FMINCON from MATLAB. FMINCON finds a constrained minimum of a function of several variables and allows the user to implement the objective gradient function.

The optimization results are shown in Table 1. The initial conditions applied are: no part variation, fixture deviation of $1 \mathrm{~mm}$ on locator $P$, and a tooling variation vector for welding guns $\mathrm{W}_{1}, \mathrm{~W}_{2}$, and $\mathrm{W}_{3}$, $\mathbf{V t}=[1.0,1.0,0.0]^{\mathrm{T}} \mathrm{mm}$. After assembly, locating fixture $P$ is released. As can be seen from the results, the final assembly variation can be reduced by $65 \%$ from the initial fixture position, $\mathbf{X}_{0}=(100,50,0)$, to the optimal position $\mathbf{X}^{*}=(72.5,0,0)$. From the solution, we can see that the optimization algorithm moves the locator $P$ further from the main source of variation, $\mathrm{W}_{1}$, while also avoiding the other locators on part 1.

\section{Conclusions}

This paper analyzes the impact of fixture position on sheet metal assembly variation and proposes a fixture design methodology to minimize assembly variation. Three sources of variation were considered: part variation, fixture variation, and welding 


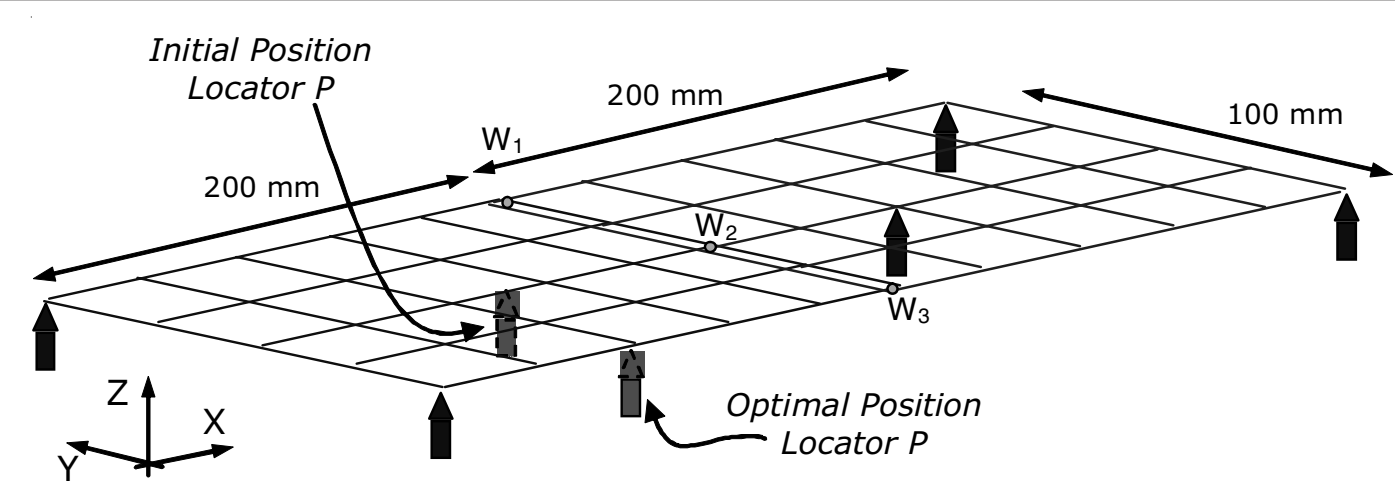

Figure 12

Locating Fixture Optimization for Two-Plate Assembly

Table 1

Application Example Results

\begin{tabular}{cccc}
\hline \multicolumn{2}{c}{ Initial Fixture Location } & \multicolumn{2}{c}{ Optimal Fixture Location } \\
\hline X Location & $\begin{array}{c}\text { Objective } \\
\text { Function }\end{array}$ & X Location & $\begin{array}{c}\text { Objective } \\
\text { Function }\end{array}$ \\
\hline $\mathrm{X}_{0}=(100,50,0)$ & $\mathrm{F}=0.2594$ & $\mathrm{X}^{*}=(72.5,0,0)$ & $\mathrm{F}=0.0901$ \\
\hline
\end{tabular}

gun variation. The assembly process is modeled based on the mechanistic simulation methodology (Liu and Hu 1997). This methodology assumes that: sheet metal deformation is in the linear elastic range; the material is isotropic; fixture and weld gun are rigid; there is no thermal deformation; and the stiffness matrix remains constant for small part deformations. An extension to the mechanistic simulation model was developed to include the effects of fixture variation and welding gun variation. With respect to the impact of fixture position on assembly variation, the following was observed:

- Part Variation: In the absence of tooling variation, fixture position has no major impact on assembly variation in the presence of part variation. The final assembly variation is only a function of part deviation. The springback effect is totally compensated by the relocation effect when fixtures are moved to different positions.

- Fixture Variation: In the presence of fixture variation, assembly variation depends on fixture positions. A general rule for variation reduction is to avoid locating non-nominal fixtures close to welding locations and other fixtures. An optimal fixture position can be found.
- Welding Gun Variation: In the presence of welding gun variation, assembly variation depends on the fixture positions. The guideline for fixture design is to move fixtures as far as possible from the locations of faulty welding gun. This minimizes any restraint to part deformation. In general, the optimal solution locates the fixtures such that they do not provide any support to the parts during the assembly process. However, this general solution is not feasible. Parts must be held or supported at a specific position before assembly.

Finally, an optimization algorithm is presented. The algorithm allows the minimization of the final assembly variation given part and tooling variation by moving fixtures (locators and clamps) to optimal positions.

\section{Acknowledgment}

The authors gratefully acknowledge the financial support provided by the General Motors Collaborative Research Laboratory and the National Science Foundation award DMI-0239244.

\section{References}

Cai, W.; Hu, S.J.; and Yuan, J.X. (1996). "Deformable sheet metal fixturing: principles, algorithms, and simulations." ASME Journal of Mfg. Science and Engg. (v118), pp318-324.

Camelio, J.; Hu, S.J.; and Ceglarek, D. (2003). "Modeling variation propagation of multi-station assembly systems with compliant parts." ASME Journal of Mechanical Design (v125, n4), pp673-681.

Ceglarek, D. and Shi, J. (1995). "Dimensional variation reduction for automotive body assembly." Mfg. Review (v8, n2), pp139-154.

Ceglarek, D. and Shi, J. (1996). "Fixture failure diagnosis for the autobody assembly using pattern recognition." Trans. of ASME, Journal of Engg. for Industry (v118, n1), pp55-66. 
Ceglarek, D. and Shi, J. (1998). "Design evaluation of sheet metal joints for dimensional integrity." Journal of Mfg. Science and Engg. - Trans. of ASME (v120, n2), pp452-460.

Choudhuri, S. and De Meter, E. (1999). "Tolerance analysis of machining fixture locators." ASME Journal of Mfg. Science and Engg. (v121), pp273-281.

Ding, Y.; Ceglarek, D.; and Shi, J. (2000). "Modeling and diagnosis of multi-stage manufacturing process: part I - state space model." Japan-USA Symp. on Flexible Automation.

Ding, Y.; Shi, J.J.; Ceglarek, D. (2002). "Diagnosability analysis of multi-station manufacturing processes." Journal of Dynamic Systems Measurement and Control - Trans. of ASME (v124, n1), pp $1-13$.

Jin, J. and Shi, J. (1999). "State space modeling of sheet metal assembly for dimensional control." Journal of Mfg. Science and Engg. - Trans. of AMSE (v121, n4), pp756-762.

Kashyap, S. and DeVries, W. (1999). "Finite element analysis and optimization in fixture design." Structural Optimization (v18), pp 193-201.

Lee, J. and Haynes, L. (1987). "Finite element analysis of flexible fixturing system.” ASME Journal of Engg. for Industry (v109), pp579-584.

Liu, S.C. and Hu, S.J. (1995). "An offset element and its applications in predicting sheet metal assembly variation." Int'l Journal of Machine Tools and Manufacture (v35, n11), pp1545-1557.

Liu, S.C. and Hu, S.J. (1997). "Variation simulation for deformable sheet metal assemblies using finite element methods." ASME Journal of Mfg. Science and Engg. (v119), pp368-374.

Mantripragada, R. and Whitney, D.E. (1999). "Modeling and controlling variation propagation in mechanical assemblies using state transition models." IEEE Trans. on Robotics and Automation (v115, n1), pp124-140.

Menassa, R. and DeVries, W. (1991). "Optimization methods applied to selecting support positions in fixture design." ASME Journal of Engg. for Industry (v113), pp412-418.

Rearick, M.; Hu, S.J.; and Wu, S.M. (1993). "Optimal fixture design for deformable sheet metal workpieces." Transactions of NAMRI/ SME (v21). Dearborn, MI: Society of Manufacturing Engineers, pp407-412.

Rong, Q.; Ceglarek, D.; Shi, J. (2000). "Dimensional fault diagnosis for compliant beam structure assemblies." Journal of Mfg. Science and Engg. - Trans. of ASME (v122, n4), pp773-780.

Sayeed, Q. and De Meter, E. (1999). "Compliance based MIP model and heuristic for support layout optimization." Int'l Journal of Production Research (v37, n6), pp1283-1301.

Vallapuzha, S.; De Meter, E.; Choudhuri, S.; and Khetan, P. (2002). "An investigation of the effectiveness of fixture layout optimization methods." Int'l Journal of Machine Tools and Manufacture (v42), pp251-253.

\section{Authors' Biographies}

Dr. Jaime Camelio is currently with A.T. Kearney, Inc. Dr. Camelio obtained his BS and MS degrees in mechanical engineering from the Catholic University of Chile in 1994 and 1995, respectively. In 2002, he received his $\mathrm{PhD}$ from the University of Michigan. His research interests are in assembly modeling, tolerance analysis, and manufacturing system design and analysis. He was the recipient of the 2001 Best Paper Award from the ASME Design Engineering Div.

$\mathrm{S}$. Jack $\mathrm{Hu}$ is currently a professor in the Dept. of Mechanical Engineering at the University of Michigan. He received his MS and PhD from the University of Michigan. His research and teaching interests are in the areas of assembly and joining, statistical methods, and manufacturing systems. Dr. Hu is a fellow of ASME and a DAAD Fellow. He has received a number of awards for his work, including a CAREER Award from NSF, the 1997 Robert Caddell Memorial Award, and the 1993 Outstanding Young Manufacturing Engineer Award from the Society of Manufacturing Engineers.

Darek Ceglarek received his $\mathrm{PhD}$ in mechanical engineering from the University of Michigan in 1994 following his diploma in production engineering from Warsaw University of Technology in 1987 . He was on the research faculty at the University of Michigan from 19952000. Currently, he is an associate professor in the industrial engineering department at the University of Wisconsin-Madison. His research interests include design, control, and diagnostics of multistage manufacturing processes; developing statistical methods driven by engineering models for quality improvement; product life cycle management (PLM); reconfigurable/reusable manufacturing systems; and modeling and causality analysis of product and process key characteristics. Dr. Ceglarek is a corresponding member of CIRP and is a member of ASME, SME, NAMRI, IIE, and INFORMS. He has received a number of awards for his work, including a $2003 \mathrm{NSF}$ CAREER Award, the 1998 Dell K. Allen Outstanding Young Manufacturing Engineer Award from the Society of Manufacturing Engineers, the 1999 Outstanding Research Scientist Award from the College of Engineering, University of Michigan. He has published more than 50 technical peer-reviewed papers in various journals and international conference proceedings. 